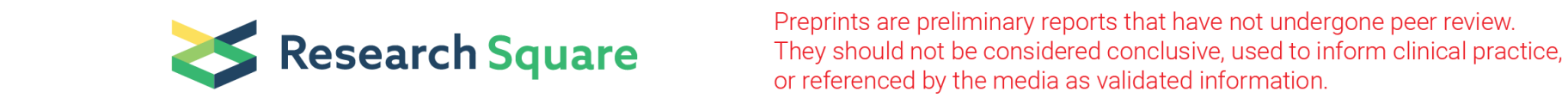

\title{
Neural Processing of Speech Sounds in ASD and First-Degree Relatives
}

\author{
Shivani P. Patel \\ Northwestern University \\ Molly Winston \\ Northwestern University \\ Janna Guilfoyle \\ Northwestern University \\ Trent Nicol \\ Northwestern University \\ Gary E. Martin \\ St. John's University \\ Nina Kraus \\ Northwestern University \\ Molly Losh ( $\sim$ m-losh@northwestern.edu ) \\ Northwest University https://orcid.org/0000-0002-9823-8249
}

\section{Research}

Keywords: autism spectrum disorder, frequency following response, pragmatic language, prosody, sound processing, auditory brainstem response Posted Date: July 29th, 2020

DOI: https://doi.org/10.21203/rs.3.rs-48454/v1

License: (c) (1) This work is licensed under a Creative Commons Attribution 4.0 International License. Read Full License 


\section{Abstract}

Background: Efficient neural encoding of sound plays a critical and widespread role in speech and language, and when impaired, may have reverberating effects on a range of communication skills. Autism spectrum disorder (ASD) is a condition involving impaired communication abilities, including atypical prosody (e.g., intonation modulation, rate, rhythm), in which atypical neural processing of speech has been implicated. Parallel patterns of communication differences have been noted in parents of individuals with ASD, who may exhibit subclinical language-related differences believed to reflect genetic liability to ASD. The present study investigated disruptions to neural processing of temporal and spectral (i.e., frequency/pitch) properties of speech sounds as a potential neurobiological mechanism underlying specific language-related impairments in ASD and related subclinical differences documented among firstdegree relatives of individuals with ASD.

Methods: Participants included individuals with ASD, their parents, and respective control groups. Group differences in temporal and spectral processing based on the neural FFR were assessed using MANCOVAs controlling for chronological age. Relationships between the FFR, pragmatic language ability, and receptive and expressive prosody skills were assessed using Pearson correlations. Familiality of the FFR in mother-child dyads were also examined using Pearson correlations.

Results: Findings revealed inefficiencies in neural encoding of speech sounds in both individuals with ASD and their parents (specifically, increased neural noise and delayed neural processing of speech sounds), as well as a less robust neural representation of spectral properties of speech sounds in individuals with ASD. Associations between neural processing of speech sounds and language-related abilities were detected in both groups, along with evidence of familiality of neural pitch processing in the ASD family groups.

Limitations: Additional investigation is necessary to determine whether relationships between FFR and pragmatic language and prosody extend to individuals with more severe language and/or cognitive impairments.

Conclusions: Overall, results suggest atypical neural processing of speech sounds may constitute an important, heritable ingredient contributing to the ASD language phenotype and subclinical phenotypic expression of genetic liability in parents.

\section{Background}

Language impairment is a hallmark of autism spectrum disorder (ASD), with pragmatic (or social) language difficulties universally observed, with broad impacts on social functioning (1-5). Contributing to such impairments are differences in prosody, which includes atypical intonation, volume modulation, and speech rate (6-9). Atypical prosody is among the first characteristics to differentiate an individuals with ASD from peers (10,11) and can significantly impact social-communicative success. Differences in auditory or speech processing have also been reported in ASD and may relate to prosodic impairments (12-15). Importantly, differences in pragmatics, prosody, and auditory processing have also been identified among first-degree relatives of individuals with ASD who are at increased genetic liability to ASD $(12,16-20)$.

The study of first-degree relatives is a powerful approach for probing fundamental, heritable features of ASD in their subclinical expression to inform the underlying biology of the complex ASD phenotype. Prior studies have shown differences among first-degree relatives of individuals with ASD in the domain of social cognition, implicating key brain regions involved in social information processing (21-28), and in a number of language-related skills. For instance, relatives of individuals with ASD show less efficient eye-voice coordination, atypical visual attention patterns during language production tasks, atypical audio-vocal integration, and broader differences in pragmatic skills, suggesting diminished fluency in aspects of language processing, similar to patterns documented in $\operatorname{ASD}(12,16,17,29,30)$

Prior work identifying relationships between prosody, auditory and speech processing, and pragmatic language in ASD demonstrates that differences in neural auditory processing impact vocal production $(12,13,31,32)$. Close associations between these language domains and neural atypicalities in individuals with ASD and their first-degree relatives suggest heritable mechanisms underlying ASD-related language impairments. Building on this work, the present study investigated neural processing of speech sounds in individuals with ASD and their biological parents, by studying the frequency following response (FFR).

The FFR is a robust neural response to sound that is highly related to speech processing and broader communication skills across the lifespan (33, 34). While based predominantly in the auditory midbrain, the FFR reflects integrated processing from the auditory periphery and central nervous system, thereby providing valuable information about neural sound processing and downstream influences on communication (35-38). Prior work using FFR in ASD has reported atypical timing and frequency encoding of speech sounds, and reduced response consistency $(14,39)$. Interestingly, atypicalities in speech sound processing have been documented using FFR in the presence of typical click-evoked sounds and normal hearing status (14, 40), suggesting that differences observed in ASD are specific to speech. A study examining neural pitch tracking of speech in ASD using FFR found decreased neural tracking of the voice pitch and reduced phase locking (41) - differences that could affect higher-level language abilities impacted in ASD, including prosody and pragmatic language $(3,5,18,42)$.

Robust associations between FFR and language skills have been demonstrated in the general population (43-45), and in language-related disorders (46-49), though little is known about how atypical FFR might relate to the profile of ASD. Russo and colleagues did not detect associations between FFRs and intellectual functioning or global measures of receptive and expressive language ability (41), but pragmatics and prosody were not examined. Given the limited work examining such key language-related correlates of FFR in ASD, it is important to examine FFR in relationship to the prosodic and pragmatic skills impacted in ASD. Examining potential links between FFR and the subclinical expression of ASD-related phenotypes in parents holds additional potential for revealing heritable, neurobiological mechanisms that can help inform the underlying etiology of ASD and its component traits. 
This study examined the hypothesis that differences in neural processing of speech sounds contribute to pragmatic and prosodic impairments in ASD, and subclinical differences among first-degree relatives. We collected FFRs to two speech stimuli in individuals with ASD, their parents, and respective control groups. Stimuli included a short speech-evoked /d $/ /$ and a longer /j『/ with ascending pitch contour, to assess neural processing of speech sounds (14, 41, 50). Responses to the $/ \mathrm{d} \nabla /$ stimulus index the timing and synchrony of neural responses. Specifically, onset of the neural response is indicated by wave $V$ and its negative trough wave $A$, while offset of the response is indicated by wave 0 . Phase locking to the fundamental frequency of the stimulus is reflected by latencies for waves $\mathrm{D}, \mathrm{E}$, and $\mathrm{F}(35,51)$. The $/ \mathrm{j} \otimes /$ stimulus provides information regarding the fidelity of neural pitch tracking. Both reflect critical components of complex speech sound processing, strongly implicated in variety of language-related disabilities, including ASD $(14,39,41,43,46,52-54)$.

Given repeated observations that parents of individuals with ASD may display subclinical pragmatic language differences $(16,17,20)$, and specifically in neural mechanisms contributing to audio-vocal integration impacting prosody (12), we predicted that the ASD parent group would display increased neural response latencies and reduced fidelity of neural pitch compared to parent controls. We predicted that atypicalities in FFR would relate to pragmatic and prosodic abilities in individuals with ASD and their parents, and that parent-child associations would emerge in the fidelity of neural response to speech sounds, which would support FFR as a potential heritable neural marker of language-related impairments in ASD.

\section{Methods}

Participants were recruited through the Northwestern University Communication Research Registry (P30DC012035), the Northwestern Child Studies Group, existing studies, and by study advertisement. Participants included 35 individuals with autism spectrum disorder (ASD group), 25 typically developing controls (ASD Control group), 49 parents of individuals with ASD (ASD Parent group), and 32 parents of typically developing individuals (Parent Control group). A subset of participants did not complete the full FFR protocol due to sensory aversions or time constraints (ASD group $n=9$ ( 3 females); ASD Control group $n=$ 1 (0 females); ASD Parent group $n=6$ (3 females); Parent Control group $n=4$ (3 females)). All participants were native English speakers with no history of hearing loss, brain injury, or presence of a known genetic condition other than ASD (e.g., fragile X syndrome). Control participants were excluded if they had first- or second-degree relatives with ASD or a history of language related impairments. All individuals with ASD had a formal diagnosis of autism or autism spectrum disorder. Diagnoses were confirmed using the Autism Diagnostic Observation Schedule-2nd Edition (ADOS-2; 55) for all participants, as well as the Autism Diagnostic Interview-Revised (ADI-R; 57). Nine individuals did not receive the ADI-R due to time limitations for testing. All parents in the ASD parent group had at least one child with ASD; however, in some cases, the child's FFR was not assessed due to various reasons (e.g., discomfort; overactivity; sensory aversions to the electrodes, etc.).

Intellectual functioning was assessed using the Wechsler Abbreviated Scale of Intelligence (WASl;58) for individuals 16 years of age or older and the Wechsler Intelligence Scale for Children-Fourth Edition (WISC-IV;59) for individuals younger than 16 years of age. See Table 1 for group comparisons of chronological age and full-scale IQ. All analyses controlled for chronological age given known differences in the frequency following response with changes in age (34, 50). 
Table 1

Group Characteristics

\begin{tabular}{|c|c|c|c|c|c|c|c|c|c|c|c|c|c|}
\hline & $\begin{array}{l}\text { ASD } \\
(n= \\
35)\end{array}$ & $\begin{array}{l}\text { ASD } \\
\text { Control } \\
(n=25)\end{array}$ & $\begin{array}{l}\text { Group } \\
\text { Comparison } \\
\text { (ASD vs. } \\
\text { ASD } \\
\text { Control) }\end{array}$ & $\begin{array}{l}\text { ASD } \\
\text { Parent } \\
(n= \\
49)\end{array}$ & $\begin{array}{l}\text { Parent } \\
\text { Control } \\
(n= \\
32)\end{array}$ & $\begin{array}{l}\text { Group } \\
\text { Comparison } \\
\text { (ASD Parent } \\
\text { vs. Parent } \\
\text { Control) }\end{array}$ & & & & & & & \\
\hline $\begin{array}{l}\text { Sex } \\
\text { Males:Females }\end{array}$ & $22: 13$ & $13: 12$ & \multicolumn{10}{|c|}{ Males:Females } & \\
\hline $\begin{array}{l}\text { Chronological } \\
\text { Age } \\
M(S D)\end{array}$ & $\begin{array}{l}16.97 \\
(6.02)\end{array}$ & $\begin{array}{l}15.16 \\
(6.21)\end{array}$ & $\begin{array}{l}t(58)=1.14 \\
p=.26\end{array}$ & $\begin{array}{l}47.14 \\
(8.50)\end{array}$ & $\begin{array}{l}43.50 \\
(7.25)\end{array}$ & $\begin{array}{l}t(73.43)= \\
2.06, p=.04\end{array}$ & & & & & & & \\
\hline $\begin{array}{l}\text { Full scale IQ } \\
M(S D)\end{array}$ & $\begin{array}{l}99.94 \\
(18.64)\end{array}$ & $\begin{array}{l}119.65 \\
(13.04)\end{array}$ & $\begin{array}{l}t(54.91)= \\
-4.70, p \\
<.01\end{array}$ & $\begin{array}{l}116.11 \\
(11.06)\end{array}$ & $\begin{array}{l}118.37 \\
(9.82)\end{array}$ & $\begin{array}{l}t(74)= \\
-0.91, p \\
=.37\end{array}$ & & & & & & & \\
\hline & \multicolumn{13}{|c|}{ ASD and ASD Control groups } \\
\hline & \multicolumn{2}{|c|}{$\begin{array}{l}\text { Discrimination/ } \\
\text { Imitation }\end{array}$} & \multicolumn{2}{|l|}{ Turn-End } & \multicolumn{2}{|l|}{ Affect } & \multicolumn{2}{|c|}{ Lexical Stress } & \multicolumn{2}{|c|}{ Phrase Stress } & \multicolumn{2}{|c|}{ Boundary } & \multirow{2}{*}{$\begin{array}{l}\text { Con } \\
\text { Stre. } \\
\text { Rec }\end{array}$} \\
\hline & $\operatorname{Rec}$ & Exp & $\operatorname{Rec}$ & Exp & $\operatorname{Rec}$ & Exp & $\operatorname{Rec}$ & Exp & $\operatorname{Rec}$ & Exp & $\operatorname{Rec}$ & Exp & \\
\hline $\begin{array}{l}\text { Response } \\
\text { Latency Wave } \\
V\end{array}$ & -0.065 & $-.491^{\star *}$ & -0.148 & -0.222 & -0.104 & 0.024 & -0.144 & -0.104 & -0.030 & -0.189 & -0.215 & 0.011 & $-0.2^{-}$ \\
\hline $\begin{array}{l}\text { Response } \\
\text { Latency Wave } \\
A\end{array}$ & 0.133 & $-.493^{\star \star}$ & -0.052 & -0.177 & -0.065 & 0.127 & -0.095 & -0.234 & 0.021 & -0.100 & 0.017 & -0.008 & -.34 \\
\hline $\begin{array}{l}\text { Response } \\
\text { Latency Wave } \\
D\end{array}$ & 0.253 & -0.143 & -0.076 & 0.001 & 0.127 & 0.136 & 0.074 & 0.079 & $0.393^{\wedge}$ & 0.199 & 0.189 & 0.158 & $-0.0^{-}$ \\
\hline $\begin{array}{l}\text { Response } \\
\text { Latency Wave } \\
\text { E }\end{array}$ & -0.099 & $-.467^{\star \star}$ & -0.087 & -0.275 & -0.130 & -0.083 & -0.262 & -0.063 & -0.050 & 0.020 & -0.339 & -0.115 & $-.3 !$ \\
\hline $\begin{array}{l}\text { Response } \\
\text { Latency Wave } \\
F\end{array}$ & 0.214 & $-.372^{*}$ & -0.023 & -0.067 & -0.053 & 0.085 & -0.053 & -0.091 & 0.341 & 0.097 & 0.089 & 0.082 & $-0.1^{*}$ \\
\hline $\begin{array}{l}\text { Response } \\
\text { Latency Wave } \\
\text { O }\end{array}$ & 0.091 & -0.031 & $.416^{*}$ & 0.133 & 0.214 & 0.072 & -0.049 & -0.039 & 0.177 & 0.013 & 0.122 & $-.438^{*}$ & 0.06 \\
\hline $\begin{array}{l}\text { Prestimulus } \\
\text { Noise }\end{array}$ & -0.044 & -0.139 & -0.174 & -0.209 & -0.033 & 0.014 & -0.030 & -0.090 & -0.024 & -0.098 & 0.121 & $0.406^{\wedge}$ & -0.0 \\
\hline $\begin{array}{l}\text { Response } \\
\text { Consistency }\end{array}$ & 0.240 & $.350^{*}$ & $0.299^{\wedge}$ & $.389^{*}$ & 0.091 & -0.177 & 0.084 & 0.245 & 0.154 & 0.252 & 0.122 & -0.077 & .431 \\
\hline $\begin{array}{l}\text { Spectral } \\
\text { Amplitude } \\
\text { (low) }\end{array}$ & 0.006 & $0.297^{\wedge}$ & $.359^{*}$ & 0.231 & -0.033 & -0.229 & -0.109 & 0.066 & -0.111 & -0.023 & 0.001 & -0.262 & 0.28 \\
\hline $\begin{array}{l}\text { Spectral } \\
\text { Amplitude } \\
\text { (mid) }\end{array}$ & 0.075 & 0.088 & $.345^{*}$ & 0.230 & -0.048 & 0.015 & -0.109 & -0.007 & -0.151 & -0.032 & -0.009 & $-0.354^{\wedge}$ & 0.22 \\
\hline /ja/ Pitch Error & 0.161 & -0.142 & 0.283 & $-.395^{*}$ & 0.176 & -0.121 & 0.110 & -0.056 & 0.117 & 0.101 & 0.240 & 0.073 & $-0.3^{\prime}$ \\
\hline $\begin{array}{l}\text { /ja/ Pitch } \\
\text { Strength }\end{array}$ & -0.038 & 0.144 & $-.405^{*}$ & $.382^{*}$ & -0.260 & 0.128 & -0.227 & -0.033 & -0.100 & 0.015 & $-.549^{*}$ & -0.343 & 0.18 \\
\hline $\begin{array}{l}\text { /ja/ } \\
\text { Correlation } \\
\text { Coefficient }\end{array}$ & $-0.391^{\wedge}$ & 0.131 & -0.279 & 0.097 & -0.296 & 0.086 & -0.178 & -0.144 & -0.297 & -0.310 & -0.182 & -0.099 & 0.11 \\
\hline
\end{tabular}

\section{Hearing Status}

Click-evoked wave $\mathrm{V}$ latencies were reviewed to determine normal hearing status. Latencies were required to be within two standard deviations of normative click-evoked latencies (34) for inclusion in this study. 


\section{Electrophysiological Recordings}

All responses were collected using the Bio-logic Navigator Pro AEP system. FFRs were collected using a vertical montage (active Cz, forehead ground, ipsilateral earlobe references) using $\mathrm{Ag}-\mathrm{AgCl}$ electrodes with impedances less than $5 \mathrm{kOhms}$. The stimuli include one $40 \mathrm{~ms}$ speech syllable / $\mathrm{d} \nabla /$ and one $230 \mathrm{~ms}$ naturally voiced / j $\varangle /$ syllable with an ascending pitch contour $(130-220 \mathrm{~Hz}$ ) applied in Praat (60). Each stimulus was presented monaurally to the right ear at $80 \mathrm{~dB}$ SPL through insert earphones (ER-3A, Etymotic Research) while participants sat in a comfortable chair in a quiet room. Stimuli were presented with alternating polarity in order to minimize stimulus artifacts. Responses with greater than $+/-35 \mu \mathrm{V}$ are rejected as artifacts, yielding a total of $6000 / \mathrm{d} \nabla /$ and $4800 / \mathrm{j} \nabla /$ responses. Participants watched a movie of their choice to maintain relaxation for the duration of the FFR collection. Response Consistency was measured using a Pearson correlation of FFRs to the /da/ stimulus. The reported values underwent a Fisher transformation to z-values. Pitch tracking of the $/ \mathrm{j} \otimes /$ responses was assessed using autocorrelation and Fast Fourier Transform techniques in MATLAB. The entire response was assessed and the greatest spectral activity between 100 and $250 \mathrm{~Hz}$ was calculated for overlapping 40-ms bins sliding in 1 ms increments. Pitch tracking ability is reflected by measures of Pitch Strength, Pitch Error, and Correlation Coefficient. Pitch Strength, which was measured via response periodicity, indicates the robustness of neural phase locking to the stimulus. Pitch Error refers to the difference in frequency between the maximal spectral energy at each time point and the corresponding time point in the stimulus and is reported in $\mathrm{Hz}$. Correlation Coefficient is a direct Pearson correlation between the known pitch of the stimulus and the participants' neural representation of the stimulus.

Prior to analysis, recordings were evaluated for validity using both objective and subjective measurements, including presence of excess prestimulus noise and recording quality. Prestimulus baseline RMS amplitude provides a measurement of subject internal noises prior to stimulus onset. Because there should not be time-locked activity during this period, recordings containing greater than $0.06 \mu \mathrm{V}$ RMS amplitude during this time region were removed. In addition, a trained rater (author: TN) blind to diagnosis reviewed remaining recordings to provide a rating of recording quality on a 4-point scale (1: worst; 4: best). Recordings that received ratings of 1 or 2 were removed prior to analyses and are not included in the sample sizes reported here.

\section{Speech And Language Correlates}

Pragmatic Language Skills. The Pragmatic Rating Scale-School Age (PRS-SA; 61) was used to assess pragmatic language skills in the ASD and ASD Control groups. The PRS-SA is rated from video recordings of semi-structured play and conversation from the ADOS-2 (55). In the ASD Parent and Parent Control groups, the Pragmatic Rating Scale (PRS; 62) was used to assess pragmatic language skills. The PRS is coded based on a semi-structured conversational interview in which an examiner asks the parent a series of questions about their childhood, schooling, social relationships, and occupation. Both the PRS-SA and PRS comprise different subscales that index similar skills. The PRS-SA subscales include: presupposition (e.g., redundant conversation, inadequate clarification, failure to provide background information); discourse management (e.g., acknowledgement, reciprocal conversation, response elaboration); speech/language behaviors that affect pragmatic language (e.g., overly formal language, scripted language, and language that is difficult to understand); suprasegmentals (e.g., intonation of voice, rate of speech, volume modulation); and nonverbal communication (e.g., use of gestures, eye-contact, and facial expressions). The PRS subscales include: dominant conversation style (e.g., too detailed, tangential); listener expectation (e.g., unable to clarify, failure to reciprocate); and suprasegmentals (e.g., intonation of voice, rate of speech, volume modulation). For both the PRS-SA and the PRS, two coders blind to group independently rated the interactions for pragmatic language features on a three-point scale, with 0 indicating absent, 1 indicating mild, and 2 indicating present. The coders resolved coding discrepancies through discussion in order to reach a consensus.

Prosodic Ability. The Profiling Elements of Prosody in Speech-Communication (PEPS-C; 63) assessed prosodic ability in all participants. The PEPS-C measures receptive and expressive prosody across eight specific skill areas, including the ability to understand and use prosody in a way that communicates a specific function, such as lexical stress or affect, as well as the ability to discriminate and imitate intonation patterns. Each domain of the PEPS-C contained 16 items and participants received one point per correct response.

\section{Statistical Analysis}

Responses were examined using a series of multivariate analyses of covariance (MANCOVA) for the /d $/$ and /j区/ stimuli to assess differences between the ASD and ASD Control groups, as well as the ASD Parent and Parent Control groups. The initial MANCOVA for the /d汶 stimulus included all latency-related variables (e.g., waves A-O, which reflect the onset and offset of the neural response as well as phase locking to the fundamental frequency). A secondary MANCOVA for the /d囚/ stimulus was conducted to assess spectral properties of the response and included measures of spectral amplitude (low and mid frequencies that correspond to the fundamental frequency (F0) and the first formant (F1), respectively). Additional univariate analyses of covariance were conducted to assess for group differences in response consistency and prestimulus noise. For the / $\mathrm{j} \otimes /$ stimulus, a MANCOVA assessing differences in pitch strength, pitch error, and correlation coefficient was conducted. Pearson correlations were conducted to assess relationships between FFR variables and pragmatic language and prosodic abilities on the PRS-SA and PRS, and PEPS-C, respectively. Planned comparisons were investigated following each MANCOVA even when the overall model was nonsignificant given our hypotheses and to guard against Type 2 error. Each of the seven domains of the PEPS-C was examined separately in correlations. For the PRS-SA and PRS, specific domain scores were investigated when associations between the FFR variable and total pragmatic language violations were detected. Correlations were conducted in the ASD and ASD Control groups combined and the ASD Parent and Parent

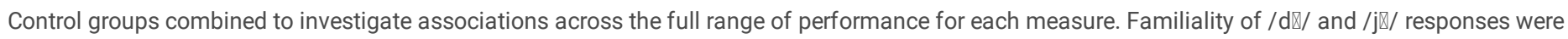
assessed using exploratory mother-child correlations (ASD dyads $n=16$; Control dyads $n=19$ ). Father-child correlations were not explored due to a limited number of father-child dyads (ASD dyads $n=4$; Control dyads $n=2$ ). To investigate whether mother-child correlations were not a by-product of similar patterns observed at the group level, Pearson correlations were conducted between unrelated mother-child dyads. For these analyses, individuals in the ASD group were randomly paired with an ASD mother and vice versa in the control group. 


\section{Results \\ Group Differences in FFR}

/d区/ Stimulus. The overall model comparing latencies between the ASD and ASD Control groups was statistically significant $(F=2.71, p=.02 ;$ see Fig. 1$)$. The ASD group exhibited significantly greater response latencies for waves $A(F=9.11, p=.005), E(F=7.55, p=.01)$, and $F(F=4.31$, $p=.04)$, and a marginally greater response latency for wave $V(F=3.64, p=.06)$. ASD and ASD Control groups did not differ in response latency for wave $D(F=.70, p=.41)$ or wave $0(F$ $=.72, \mathrm{p}=.40)$. The model comparing spectral amplitude between the ASD and ASD Control groups was not statistically significant $(F=1.94, p=.15)$. The ASD group exhibited significantly greater prestimulus noise $(F=7.53, p=.006)$ and reduced response consistency $(F=25.74, p<.001)$ compared to controls.

The overall model comparing latencies between the ASD Parent and Parent Control groups approached statistical significance $(F=2.11, p=.06)$. The ASD Parent group exhibited significantly greater response latencies for waves $V(F=4.77, p=.03)$ and $A(F=10.06, p=.002 ;$ see $F i g$. 1). ASD Parent and Parent Control groups did not differ in response latencies for waves $D(F=0.05, p=.83), E(F=2.14, p=.15)$, or $F(F=1.52, p=.22)$, and $O(F=0.27, p=.61)$. Comparison of spectral amplitude between the ASD Parent and Parent Control groups was not statistically significant $(F=1.58, p=.21)$, and there were no differences in prestimulus noise $(F=0.01, p=.94)$. Results revealed marginally poorer response consistency $(F=3.45, p=.07)$ in the ASD Parent group.

/j区/ Stimulus. The model assessing pitch tracking in the ASD and ASD Control groups approached significance $(F=2.14, p=.11)$. The ASD group exhibited reduced pitch strength compared to controls $(F=6.16, p=.02)$. Groups did not differ in pitch error $(F=2.51, p=.12)$ or correlation coefficient $(F=1.47, p=.23)$.

The overall model assessing pitch tracking in the ASD Parent and Parent Control groups did not reach statistical significance $(F=0.55, p=.65)$.

\section{Speech And Language Correlates Of Ffr}

Pragmatic Language in ASD and ASD Control groups. In ASD and ASD Control groups combined, increased pragmatic language violations were associated with a longer latency for wave $\mathrm{E}(r=.33, p=.02)$, increased prestimulus noise $(r=.36, p=.008)$, decreased response consistency $(r=-.52, p<.001)$, increased pitch error $(r=.34, p=.02)$, and reduced pitch strength $(r=-.42, p<.01)$. Each of these FFR variables, except latency for wave E, were associated with increased difficulty with discourse management (e.g., topic initiation, interrupting; $r s>1.29$ /, ps $\leq .05$ ). Latency for wave $E$, prestimulus noise, and response consistency, were also related to impairments in nonverbal communication (e.g., atypical eye contact, gestures; $r s>\mid .28 /$, $p s<.05$ ). Response consistency and pitch strength were additionally related to increased violations in the speech/language behaviors domain (e.g., overly formal speech; stereotyped utterances; rs $>$ /.32/, $p s$ $<$.05). Longer latencies for waves $A$ and $E$, as well as reduced response consistency, greater pitch error, and decreased pitch strength were associated with increased suprasegmental difficulties (e.g., intonation modulation, speech rate; $r s>|.28|$, $p s<.05$ ).

Pragmatic Language in ASD Parent and Parent Control groups. In the parent groups collapsed, increased pragmatic language violations were associated with decreased spectral amplitude for the fundamental frequency $(r=-.26, p=.04)$ and less response consistency $(r=-.26, p=.04)$. Associations with spectral amplitude for the fundamental frequency were detected with dominant conversational style (e.g., tangential comments, topic preoccupation; $r=-30, p=.02$ ) and pragmatic language violations related to listener expectations (e.g., fails to reciprocate, vague; $r=-.27, p=.04$ ). The relationship between response consistency and pragmatic language violations appeared to be driven by differences in suprasegmentals $(r=-.35, p<.01)$.

Prosodic Ability. In the ASD and ASD control groups, associations with receptive prosody skills emerged, with increased neural response latency and reduced response consistency associated with poorer Contrastive Stress. Sporadic associations were observed between measures of response latency, spectral amplitude, and pitch tracking with Turn-End and Boundary understanding. Poorer expressive prosody, particularly in the domains of Imitation, Turn-End, and Boundary expression, was associated with increased neural response latency and reduced response consistency, as well as poorer pitch tracking. Affect, Lexical Stress, and Phrase Stress domains were not associated with FFR (see Table 2).

Similar to patterns identified in ASD and ASD Control groups, in parent groups, poorer receptive prosody skills in the domain of Contrastive Stress were associated with increased neural response latencies and reduced response consistency, as well as reduced spectral amplitude of the fundamental frequency. Poorer expressive prosody skills in the domain of Contrastive Stress were associated with reduced spectral amplitude of the fundamental frequency. Sporadic associations between Phrase Stress and Boundary expression and neural response latencies emerged. Similar to findings in the ASD and ASD Control groups, several domains of prosody were not related to FFR (see Table 3).

\section{Familiality Of Ffr}

For mother-child ASD dyads, response latencies for wave $D(r=.52, p=.04)$ and prestimulus noise $(r=.52, p=.04)$ were positively correlated. Further, pitch error $(r=-.74, p<.01)$ and pitch strength $(r=-.72, p<.001)$ were negatively correlated.

In mother-child Control dyads, responses latencies for waves $\mathrm{V}(r=.64, p<.01)$ and $\mathrm{A}(\mathrm{r}=.63 \mathrm{p}<.01)$ were positively associated. Additionally, spectral amplitude for the first formant frequency was positively associated $(r=.60, p<.01)$. No significant correlations were evident in the mismatched mother-child dyads in ASD nor ASD Control families $(p>.25)$.

\section{Discussion}


This study examined FFR as a potential heritable, neural mechanism contributing to the ASD language phenotype, and its more subtle expression in relatives, who are at increased genetic liability to ASD. Consistent with hypotheses, both the ASD and ASD Parent groups showed diminished FFR to complex speech sounds, with more pervasive differences evident in the ASD group. Indices of poorer FFR along several key variables were related to elevated pragmatic language differences, and poorer expressive prosody skills across groups. Evidence of familiality of FFR was also detected. Together, these findings point towards disruptions in neural processing of speech sounds as a heritable neurobiological mechanism in ASD that may contribute to the complex ASD language profile.

Importantly, findings revealed overlapping FFR differences in prestimulus noise and response latencies among individuals with ASD and their parents, suggesting that altered temporal processing of speech sounds is influenced by genetic predisposition to ASD. Results revealed delayed onset of neural processing of the speech syllable / $\mathrm{d} \mathbb{\mathrm { d }} /$, in both individuals with ASD and their parents. The ASD group also exhibited increased neural response latencies for waves representing the acoustic properties of the stimulus, suggesting decreased phase locking to the stimulus frequency. These delays indicate increased neural conduction time in both individuals with ASD and their parents, which may be a byproduct of increased prestimulus noise, which can influence sensory encoding (64-67). Indeed, prior work has shown that children affected by linguistic deprivation exhibit greater neural noise. By contrast, collegiate athletes demonstrate an improved ability to minimize neural noise to more clearly tune into the speech signal, further highlighting neural noise as a potentially key indicator of general auditory neural acuity $(68,69)$. Furthermore, decreased response consistency in the ASD group suggests greater variability in speech sound representations. Such disruptions are believed to play a role in impaired phonological development (e.g., formation and use of speech sounds) in individuals with reading difficulties (54), and could contribute to inefficient neural processing observed in ASD.

Consistent with prior findings demonstrating reduced pitch tracking in individuals with ASD (41), results indicated reduced pitch strength to the /ja/ stimulus, but not increased pitch error, in the ASD group. The lack of differences in pitch processing among ASD parents is not necessarily surprising, given that parents do not show clinical impairments. Rather, the attenuated FFR differences in parents may be notable in implicating a refined constellation of neural processing abilities specifically influenced by ASD genetic liability (namely, temporal and spectral processing) and not encumbered by the influence of comorbidities or multiply impaired symptom domains typical in ASD. Significant parent-child associations in temporal processing of speech sounds further suggest specific FFR components that might constitute potent, heritable markers of neural differences related to the language profile in ASD.

Evidence implicating atypical FFR as a neural mechanism contributing to the ASD language phenotype is further supported by associations between FFR and pragmatic language and prosodic skills across groups. Specifically, significant, parallel relationships across the ASD, ASD parent, and control groups were detected between neural response timing and pitch representations and increased pragmatic language violations, particularly in the area of suprasegmentals. Given that pragmatic impairment is a defining feature of ASD (1-5), and subclinical pragmatic language differences have been repeatedly documented in ASD relatives $(12,16-18,20)$, associations between pragmatics and neural processing of speech sounds are significant in implicating FFR as neural mechanism related to a core symptom domain in ASD. Associations between response latencies and key pragmatic language skills in parents are also intriguing in suggesting that even subtle differences in neural processing of sound might have reverberating effects on downstream, more complex language abilities, such as pragmatics, which rely on the integration of many foundational mechanisms and skills. This is consistent with prior evidence of relationships between neural response latencies and magnitudes with overall cognitive and language abilities (41), and implicates FFR as a potentially important target for study in understanding the complex brain basis of pragmatic language impairments that characterize ASD and their subclinical manifestation in relatives.

Relationships between FFR and prosodic abilities were also detected, but in less clear or robust patterns than in pragmatics. Poorer contrastive stress understanding was consistently related to increased neural response latencies and reduced response consistency across groups, suggesting that receptive prosody is an important aspect of communication impacted by inefficient neural processing of speech sounds. Furthermore, relationships between poorer temporal processing and pitch tracking, as well as reduced response consistency, and increased expressive prosodic errors in the ASD and ASD Control groups may reflect a greater impact of inefficient neural processing of speech sounds on prosodic production. However, FFR associations with other prosodic skills were not clearly evident. In parents, this is perhaps unsurprising, considering the near-ceiling effects on the PEPS-C demonstrated by parent groups. Inconsistent findings more generally may have related to specific features of the task. For instance, receptive prosody tasks were not timed, so the counterintuitive relationships that emerged (e.g., reduced pitch strength associated with greater turn-end understanding) could reflect more effortful processing, which yielded successful behavioral responses on the PEPS-C despite delayed neural processing of speech sounds detected in the ASD and ASD Control groups. Inconsistent findings could also constitute false discovery resulting from multiple tests conducted, in our effort to uncover potential relationships between FFR and prosodic skills. Relationships that did emerged were of medium effect sizes and thus provide some preliminary evidence of a link between temporal and spectral processing at the neural level and clinically meaningful deficits in prosody that will be important to investigate further with larger samples, and a wider array of more tightly constrained prosodic assessments that more sensitively tap prosodic variability across clinically affected and unaffected groups.

\section{Limitations}

Several limitations should be considered in interpreting results. First, the study's focus on verbally fluent individuals with ASD, while important for reducing comorbidities, may limit generalization of findings to clinically affected individuals with lower levels of language abilities. Therefore, it will be important for future research to investigate whether differences in FFR, and relationships between FFR and pragmatic language and prosody extend to individuals with more severe language and/or cognitive impairments. Likewise, the age range of individuals with ASD did not span the lifespan, so it is unclear if the FFR serves as a biomarker early in development as well as later in adulthood. There is cross-sectional evidence to suggest that the FFR changes across the lifespan, so it is possible group differences may be less or more robust at certain times in development. Finally, familiality of FFR was only assessed with mother-child dyads due to the limited number of fathers. Some evidence indicates differences between maternal vs. paternal traits and ASD symptomatology in their children, suggesting potential differences in familiality between mothers and fathers $(30,70,71)$, making it important to further study patterns of lineality to investigate the inheritance of FFR and provide insights into gene-brain-behavior connections. 


\section{Conclusions}

This study demonstrated converging evidence of differences in FFR in ASD and parents, familiality of FFR, and associations between FFR and pragmatics and prosody. These findings add to the understanding of neurobiological contributions to speech and language deficits characteristic of ASD and implicate the FFR as a potentially heritable neurobiological marker of language-related deficits in ASD. Moreover, parallel findings in parents of individuals with ASD as well as associations with language abilities suggest that the FFR is impacted across the spectrum of genetic vulnerability to ASD, including relatives who do not exhibit clinical impairments. Given existing research demonstrating the experience-related malleability of neural responses to speech (35, 72-75), the relationships detected between the FFR and pragmatic language and prosody in the present work may also support the study of FFRs to speech sounds as a sensitive, biological index of response to speech and language intervention.

\section{Abbreviations}

ASD

autism spectrum disorder

FFR

frequency following response

ADOS

Autism Diagnostic Observation Schedule, 2nd Edition

PRS-SA

Pragmatic Rating Scale-School

PRS

Pragmatic Rating

PEPS-C

Profiling Elements of Prosody in Speech-Communication

\section{Declarations}

\section{Ethics Approval and Consent to Participate.}

Study procedures were approved by the Northwestern University Institutional Review Board (IRB). Adult participants provided written consent and participants under the age of 18 or whose parents maintained guardianship were consented via parent consent and assented.

\section{Consent for Publication.}

Not applicable.

\section{Availability of Data and Materials.}

Data will be made available through the National Institute of Mental Health Data Archive (NDA) formerly the National Database for Autism Research (NDAR) at the completion of the funding period.

\section{Competing Interests.}

NK has equity interest in Synaural, Inc., a company working to develop a user-friendly measure of auditory processing. Authors SPP, MW, JG, TN, GEM, and ML declare that they have no competing interests.

\section{Funding.}

This research was supported by the National Institutes of Health (R01DC010191, R03MH107834, P30DC012035) and the National Science Foundation (DGE1324585)

\section{Authors' Contributions.}

ML conceived the study and oversaw all data collection, analyses, and manuscript preparation. SP, MW, JG, and TN assisted in data acquisition, processing, and analysis, and contributed to manuscript preparation. GEM and NK assisted with data interpretation and manuscript preparation. All authors read and approved the final manuscript.

\section{Acknowledgements.}

The authors are grateful to the individuals and families who participated in this research.

\section{References}

1. Baltaxe CAM, Simmons JQ. Bedtime soliloquies and linguistic competence in autism. J Speech Hear Disord. 1977;42(3):376-93.

2. Landa R. Social language use in Asperger syndrome and high-functioning autism. Asperger Syndr. 2000;125-55. 
3. Losh M, Martin GE, Klusek J, Hogan-Brown AL. Pragmatic Language in autism and fragile X syndrome: genetic and clinical applications. Perspect Lang Learn Educ. 2012;19(2):48-55.

4. Peppé S, McCann J, Gibbon F, O’Hare A, Rutherford M. Assessing prosodic and pragmatic ability in children with high-functioning autism. J Pragmat. 2006;38(10):1776-91.

5. Tager-Flusberg H, Edelson L, Luyster R. Language and communication in autism spectrum disorders. Autism Spectr Disord. 2011;172-85.

6. Diehl JJ, Paul R. Acoustic differences in the imitation of prosodic patterns in children with autism spectrum disorders. Res Autism Spectr Disord. 2012;6(1):123-34.

7. McCann J, Peppé S. Prosody in autism spectrum disorders: a critical review. Int J Lang Commun Disord. 2003;38(4):325-50.

8. Paul R, Shriberg LD, McSweeny J, Cicchetti D, Klin A, Volkmar F. Brief report: Relations between prosodic performance and communication and socialization ratings in high functioning speakers with autism spectrum disorders. J Autism Dev Disord. 2005;35(6):861.

9. Shriberg LD, Paul R, McSweeny JL, Klin A, Cohen DJ, Volkmar FR. Speech and prosody characteristics of adolescents and adults with high-functioning autism and Asperger syndrome. J Speech, Lang Hear Res. 2001.

10. Mesibov GB. Treatment issues with high-functioning adolescents and adults with autism. In: High-functioning individuals with autism. Springer; 1992. p. $143-55$.

11. Van Bourgondien ME, Woods AV. Vocational possibilities for high-functioning adults with autism. In: High-functioning individuals with autism. Springer; 1992. p. 227-39.

12. Patel SP, Kim JH, Larson CR, Losh M. Mechanisms of voice control related to prosody in autism spectrum disorder and first-degree relatives. Autism Res. 2019;12(8):1192-210.

13. Russo N, Larson C, Kraus N. Audio-vocal system regulation in children with autism spectrum disorders. Exp Brain Res. 2008;188(1):111-24.

14. Russo N, Nicol T, Trommer B, Zecker S, Kraus N. Brainstem transcription of speech is disrupted in children with autism spectrum disorders. Dev Sci. 2009;12(4):557-67.

15. Russo NM. A key to understanding social communication deficits in autism spectrum disorders: Neural processing of sound and speech intonation. Northwestern University; 2008.

16. Landa R, Piven J, Wzorek MM, Gayle JO, Chase GA, Folstein SE. Preliminary communication social language use in parents of autistic individuals. Psychol Med. 1992;22(1):245-54.

17. Losh M, Childress D, Lam K, Piven J. Defining key features of the broad autism phenotype: A comparison across parents of multiple-and single-incidence autism families. Am J Med Genet Part B Neuropsychiatr Genet. 2008;147(4):424-33.

18. Patel SP, Nayar K, Martin GE, Franich K, Crawford S, Diehl JJ, et al. An Acoustic Characterization of Prosodic Differences in Autism Spectrum Disorder and First-Degree Relatives. J Autism Dev Disord. 2020;1-14.

19. Paul R, Orlovski SM, Marcinko HC, Volkmar F. Conversational behaviors in youth with high-functioning ASD and Asperger syndrome. J Autism Dev Disord. 2009;39(1):115-25.

20. Piven J, Palmer P, Jacobi D, Childress D, Arndt S. Broader autism phenotype: evidence from a family history study of multiple-incidence autism families. Am J Psychiatry. 1997;154(2):185-90.

21. Adolphs R, Spezio ML, Parlier M, Piven J. Distinct face-processing strategies in parents of autistic children. Curr Biol. 2008;18(14):1090-3.

22. Losh M, Adolphs R, Poe MD, Couture S, Penn D, Baranek GT, et al. Neuropsychological profile of autism and the broad autism phenotype. Arch Gen Psychiatry. 2009;66(5):518-26.

23. Losh M, Piven J. Social-cognition and the broad autism phenotype: Identifying genetically meaningful phenotypes. J Child Psychol Psychiatry. 2007;48(1):105-12.

24. Sasson NJ, Nowlin RB, Pinkham AE. Social cognition, social skill, and the broad autism phenotype. Autism. 2013;17(6):655-67.

25. Baron-Cohen S, Ring H, Chitnis X, Wheelwright S, Gregory L, Williams S, et al. fMRI of parents of children with Asperger Syndrome: a pilot study. Brain Cogn. 2006;61(1):122-30.

26. Billeci L, Calderoni S, Conti E, Gesi C, Carmassi C, Dell'Osso L, et al. The broad autism (endo) phenotype: neurostructural and neurofunctional correlates in parents of individuals with autism spectrum disorders. Front Neurosci. 2016;10:346.

27. Palmen SJMC, POL HEH, Kemner C, Schnack HG, Sitskoorn MM, Appels MCM, et al. Brain anatomy in non-affected parents of autistic probands: a MRI study. Psychol Med. 2005;35(10):1411-20.

28. Yucel GH, Belger A, Bizzell J, Parlier M, Adolphs R, Piven J. Abnormal neural activation to faces in the parents of children with autism. Cereb Cortex. 2015;25(12):4653-66.

29. Hogan-Brown AL, Hoedemaker RS, Gordon PC, Losh M. Eye-voice span during rapid automatized naming: evidence of reduced automaticity in individuals with autism spectrum disorder and their siblings. J Neurodev Disord. 2014;6(1):33.

30. Nayar K, Gordon PC, Martin GE, Hogan AL, La Valle C, McKinney W, et al. Links between looking and speaking in autism and first-degree relatives: insights into the expression of genetic liability to autism. Mol Autism. 2018;9(1):51.

31. Chen SH, Liu H, Xu Y, Larson CR. Voice F 0 responses to pitch-shifted voice feedback during English speech. J Acoust Soc Am. 2007;121(2):1157-63.

32. Liu P, Chen Z, Larson CR, Huang D, Liu H. Auditory feedback control of voice fundamental frequency in school children. J Acoust Soc Am. 2010;128(3):1306-12. 
33. Rosenhall ULF, Björkman G, Pedersen K, Kall A. Brain-stem auditory evoked potentials in different age groups. Electroencephalogr Clin Neurophysiol Potentials Sect. 1985;62(6):426-30.

34. Skoe E, Krizman J, Anderson S, Kraus N. Stability and plasticity of auditory brainstem function across the lifespan. Cereb Cortex. 2015;25(6):1415-26.

35. Chandrasekaran B, Kraus N. The scalp-recorded brainstem response to speech: Neural origins and plasticity. Psychophysiology. 2010;47(2):236-46.

36. Malmierca MS. Auditory system. In: The rat nervous system. Elsevier; 2015. p. 865-946.

37. Malmierca MS, Ryugo DK. Descending connections of auditory cortex to the midbrain and brain stem. In: The auditory cortex. Springer; 2011 . p. 189-208.

38. Sohmer H, Bauberger-Tell L, Feinmesser M, Edelstein E. Cochlear, brain stem, and cortical evoked responses in nonorganic hearing loss. Ann Otol Rhinol Laryngol. 1977;86(2):227-34.

39. Otto-Meyer S, Krizman J, White-Schwoch T, Kraus N. Children with autism spectrum disorder have unstable neural responses to sound. Exp brain Res. 2018;236(3):733-43.

40. Klin A. Auditory brainstem responses in autism: brainstem dysfunction or peripheral hearing loss? J Autism Dev Disord. 1993;23(1):15-35.

41. Russo S, Trommer, Nicol, Zecker B, et al. Deficient brainstem encoding of pitch in children with autism spectrum disorders. Clin Neurophysiol. 2008;119(8):1720-31.

42. Paul R, Augustyn A, Klin A, Volkmar FR. Perception and production of prosody by speakers with autism spectrum disorders. J Autism Dev Disord. 2005;35(2):205-20.

43. Benasich AA, Tallal P. Infant discrimination of rapid auditory cues predicts later language impairment. Behav Brain Res. 2002;136(1):31-49.

44. Krishnan A, Xu Y, Gandour J, Cariani P. Encoding of pitch in the human brainstem is sensitive to language experience. Cogn Brain Res. 2005;25(1):161-8.

45. Krizman J, Marian V, Shook A, Skoe E, Kraus N. Subcortical encoding of sound is enhanced in bilinguals and relates to executive function advantages. Proc Natl Acad Sci. 2012;109(20):7877-81.

46. Hornickel J, Anderson S, Skoe E, Yi H-G, Kraus N. Subcortical representation of speech fine structure relates to reading ability. Neuroreport. 2012;23(1):6.

47. Hornickel J, Skoe E, Kraus N. Subcortical laterality of speech encoding. Audiol Neurotol. 2009;14(3):198-207.

48. Thomson JM, Goswami U. Rhythmic processing in children with developmental dyslexia: auditory and motor rhythms link to reading and spelling. $J$ Physiol. 2008;102(1-3):120-9.

49. Banai K, Abrams D, Kraus N. Sensory-based learning disability: Insights from brainstem processing of speech sounds. Int J Audiol. 2007;46(9):524-32.

50. Bonacina S, Otto-Meyer S, Krizman J, White-Schwoch T, Nicol T, Kraus N. Stable auditory processing underlies phonological awareness in typically developing preschoolers. Brain Lang. 2019;197:104664.

51. Krizman J, Bonacina S, Kraus N. Sex differences in subcortical auditory processing emerge across development. Hear Res. 2019;380:166-74.

52. Anderson S, Kraus N. The potential role of the cABR in assessment and management of hearing impairment. Int J Otolaryngol. $2013 ; 2013$.

53. Chandrasekaran B, Hornickel J, Skoe E, Nicol T, Kraus N. Context-dependent encoding in the human auditory brainstem relates to hearing speech in noise: implications for developmental dyslexia. Neuron. 2009;64(3):311-9.

54. Hornickel J, Kraus N. Unstable representation of sound: a biological marker of dyslexia. J Neurosci. 2013;33(8):3500-4.

55. Lord C, Rutter M, DiLavore P, Risi S, Gotham K, Bishop S. Autism diagnostic observation schedule-2nd edition (ADOS-2). Los Angeles, CA West Psychol Corp. 2012.

56. Lord C, Rutter M, Le Couteur A. Autism Diagnostic Interview-Revised: a revised version of a diagnostic interview for caregivers of individuals with possible pervasive developmental disorders. J Autism Dev Disord. 1994;24(5):659-85.

57. Rutter M, Le Couteur A, Lord C. Autism diagnostic interview-revised. Los Angeles. CA West Psychol Serv. 2003;29:30.

58. Wechsler D. Manual for the Wechsler abbreviated intelligence scale (WASI). San Antonio, TX Psychol Corp. 1999.

59. Wechsler D. WISC-IV: Administration and scoring manual. Psychological Corporation; 2003.

60. Boersma P. Praat, a system for doing phonetics by computer. Glot Int. 2001;5(9):341-5.

61. Landa R. Pragmatic rating scale for school-age children. Unpubl manuscript. Balt: Kennedy Kreiger Institute; 2011.

62. Landa R. Pragmatic rating scale. Encycl autism Spectr Disord. 2013;2327-31.

63. Peppé S, McCann J. Profiling elements of prosody in speech-communication (PEPS-C). Available at Last accessed. 2015;8.

64. lemi L, Busch NA, Laudini A, Haegens S, Samaha J, Villringer A, et al. Multiple mechanisms link prestimulus neural oscillations to sensory responses. Elife. 2019;8:e43620.

65. McNair SW, Kayser SJ, Kayser C. Consistent pre-stimulus influences on auditory perception across the lifespan. Neuroimage. 2019;186:22-32.

66. Samaha J, lemi L, Postle BR. Prestimulus alpha-band power biases visual discrimination confidence, but not accuracy. Conscious Cogn. 2017;54:47-55.

67. Samaha J, Postle BR. The speed of alpha-band oscillations predicts the temporal resolution of visual perception. Curr Biol. 2015;25(22):2985-90.

68. Skoe E, Krizman J, Kraus N. The impoverished brain: disparities in maternal education affect the neural response to sound. J Neurosci. 2013;33(44):17221-31.

69. Krizman J, Lindley T, Bonacina S, Colegrove D, White-Schwoch T, Kraus N. Play Sports for a Quieter Brain: Evidence From Division I Collegiate Athletes. Sports Health. 2020;12(2):154-8.

70. Klusek J, Losh M, Martin GE. Sex differences and within-family associations in the broad autism phenotype. Autism. 2014;18(2):106-16.

71. Maxwell CR, Parish-Morris J, Hsin O, Bush JC, Schultz RT. The broad autism phenotype predicts child functioning in autism spectrum disorders. J Neurodev Disord. 2013;5(1):25. 
72. Fujioka T, Trainor LJ, Ross B, Kakigi R, Pantev C. Musical training enhances automatic encoding of melodic contour and interval structure. J Cogn Neurosci. 2004;16(6):1010-21.

73. Kraus N, Slater J, Thompson EC, Hornickel J, Strait DL, Nicol T, et al. Music enrichment programs improve the neural encoding of speech in at-risk children. J Neurosci. 2014;34(36):11913-8.

74. Song JH, Skoe E, Wong PCM, Kraus N. Plasticity in the adult human auditory brainstem following short-term linguistic training. J Cogn Neurosci. 2008;20(10):1892-902.

75. Wong PCM, Skoe E, Russo NM, Dees T, Kraus N. Musical experience shapes human brainstem encoding of linguistic pitch patterns. Nat Neurosci. 2007;10(4):420-2.

\section{Figures}
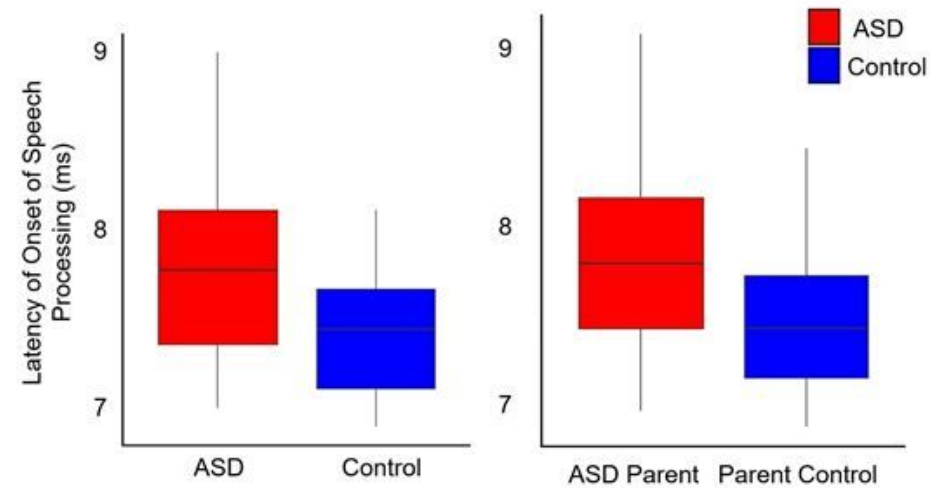

\section{Figure 1}

Latency of the onset of speech processing (ms), as represented by wave A in the ASD Group, ASD Parent Group, and respective control groups.
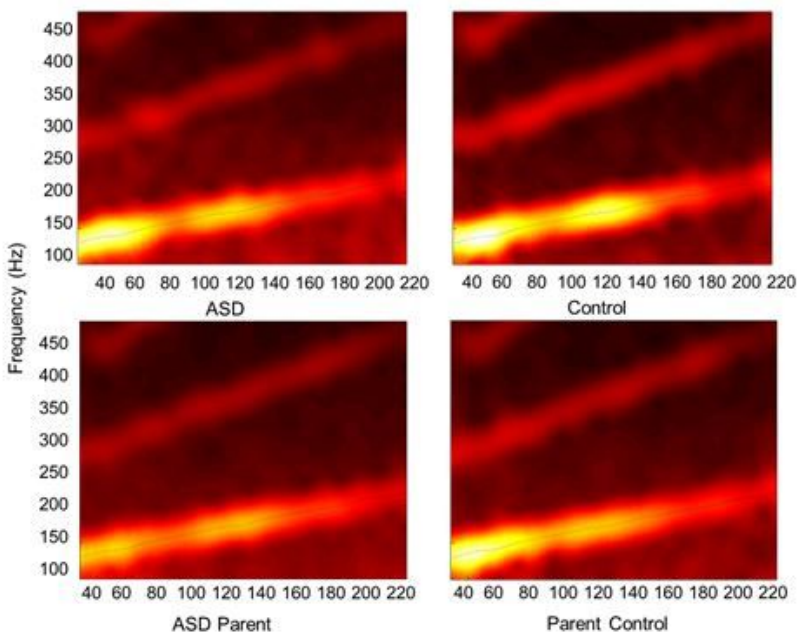

\section{Figure 2}

Neural representation of the [j『] speech-stimulus. Lighter and brighter colors represent increased fidelity in neural representation of the pitch features of the stimulus. The black dotted line signified the pitch the stimulus.

\section{Supplementary Files}

This is a list of supplementary files associated with this preprint. Click to download.

- FFRinASDParentsMolecAutismCoverLetter.docx 